\title{
GEOMETRIC VERSUS SPECTRAL CONVERGENCE FOR THE NEUMANN LAPLACIAN UNDER EXTERIOR PERTURBATIONS OF THE DOMAIN
}

\author{
JOSÉ M. ARRIETA* AND DAVID KREJČIŘÍK ${ }^{\dagger}$
}

\begin{abstract}
We analyze the behavior of the eigenvalues and eigenfunctions of the Laplace operator with homogeneous Neumann boundary conditions when the domain is perturbed. We show that if $\Omega_{0} \subset \Omega_{\epsilon}$ are bounded domains (although not necessarily uniformly bounded) and we know that the eigenvalues and eigenfunctions with Neumann boundary condition in $\Omega_{\epsilon}$ converge to the ones in $\Omega_{0}$, then necessarily we have that $\left|\Omega_{\epsilon} \backslash \Omega_{0}\right| \rightarrow 0$ while it is not necessarily true that $\operatorname{dist}\left(\Omega_{\epsilon}, \Omega_{0}\right) \stackrel{\epsilon \rightarrow 0}{\longrightarrow} 0$. As a matter of fact we will construct an example of a perturbation where the spectra behave continuously but $\operatorname{dist}\left(\Omega_{\epsilon}, \Omega_{0}\right) \stackrel{\epsilon \rightarrow 0}{\longrightarrow}+\infty$.
\end{abstract}

\section{INTRODUCTION}

This paper is concerned with the behavior of the eigenvalues and eigenfunctions of the Laplace operator in bounded domains when the domain undergoes a perturbation. It is well known that if the boundary condition that we are imposing is of Dirichlet type, the kind of perturbations that we may allow in order to obtain the continuity of the spectra is much broader than in the case of Neumann boundary condition. This is explicitly stated in the pioneer work of Courant and Hilbert [5] and it has been subsequently clarified in many works, see [4, 2, 6] and reference therein among others. See also [8] for a general text on different properties of eigenvalues and [9] for a study on the behavior of eigenvalues and in general partial differential equations when the domain is perturbed.

In particular, with Dirichlet boundary condition we may consider the case where the fixed domain is a bounded "smooth" domain $\Omega_{0} \subset \mathbb{R}^{N}, N \geq 2$, and the perturbed domain is $\Omega_{\epsilon}$ in such a way that $\Omega_{0} \subset \Omega_{\epsilon}$, that is we consider exterior perturbation of the domain. We may have perturbations of this type where $\left|\Omega_{\epsilon} \backslash \Omega_{0}\right| \geq \eta$ for some fixed $\eta>0$ and still we have the convergence of the eigenvalues and eigenfunctions. Moreover, we may even have the case $\left|\Omega_{\epsilon} \backslash \Omega_{0}\right| \rightarrow+\infty$ and still we have the convergence of the eigenvalues and eigenfunctions.

Date: 29 January 2009.

*Partially supported by Grants PHB2006-003-PC and MTM2006-08262 from MEC and by "Programa de Financiación de Grupos de Investigación UCM-Comunidad de Madrid CCG07-UCM/ESP-2393. Grupo 920894" and SIMUMAT-Comunidad de Madrid, Spain .

${ }^{\dagger}$ Partially supported by the Czech Ministry of Education, Youth and Sports within the project LC06002. 
To obtain and example of this situation is not too difficult. If we consider for instance $\Omega \subset \mathbb{R}^{2}$, given by $\Omega_{0}=(0,1) \times(-1,0)$ and

$$
\Omega_{\epsilon}(a)=\{(x, y): 0<x<1,-1<y<a(1+\sin (x / \epsilon))\} \supset \Omega_{0}
$$

where $a>0$ is fixed, we can easily see that the eigenvalues and eigenfunctions of the Laplace operator with Dirichlet boundary condition in $\Omega_{\epsilon}$ converge to the ones in $\Omega_{0}$. Moreover $\left|\Omega_{\epsilon}\right|=\left|\Omega_{0}\right|+\int_{0}^{1} a(1+\sin (x / \epsilon)) d x \sim\left|\Omega_{0}\right|+a$ for $\epsilon$ small enough. Moreover, it is not difficult to modify the example above choosing the constant $a$ dependent with respect to $\epsilon$ in such a way that $a(\epsilon) \rightarrow+\infty$ and such that we still get that the eigenvalues and eigenfunctions in $\Omega_{\epsilon}(a(\epsilon))$ converge to the ones in $\Omega_{0}$ and $\left|\Omega_{\epsilon}(a(\epsilon)) \backslash \Omega_{0}\right| \rightarrow+\infty$. This example shows that the class of perturbations that we may allow to get the "spectral convergence" of the Dirichlet Laplacian is very broad and that knowing that the eigenvalues and eigenfunctions of the Dirichlet Laplacian converge does not have many "geometrical" restrictions for the domains.

The case of Neumann boundary condition is much more subtle. As a matter of fact, for the situation depicted above it is not true that the spectra converge. So we ask ourselves the following questions: if we have a domain $\Omega_{0}$ and consider a perturbation of it given by $\Omega_{0} \subset \Omega_{\epsilon}$, where we assume that all the domains are smooth and bounded although not necessarily uniformly bounded on the parameter $\epsilon$, then if we have the convergence of the eigenvalues and eigenfunctions,

(Q1) should it be true that $\left|\Omega_{\epsilon} \backslash \Omega_{0}\right| \stackrel{\epsilon \rightarrow 0}{\longrightarrow} 0$ ?

(Q2) should it be true that $\operatorname{dist}\left(\Omega_{\epsilon}, \Omega_{0}\right)=\sup _{x \in \Omega_{\epsilon}} \operatorname{dist}\left(x, \Omega_{0}\right) \stackrel{\epsilon \rightarrow 0}{\longrightarrow} 0$ ?

We will see that the answer to the first question is Yes and, surprisingly, the answer to the second one is No.

Observe that, as the example above shows, the answer to both questions for the case of Dirichlet boundary condition is No.

In Section 2 we recall a result from [1, 3] which provides a necessary and sufficient condition for the convergence of eigenvalues and eigenfunctions when the domain is perturbed. In Section 3 we provide an answer to question (Q1) and in Section 4 we provide an answer to question (Q2).

\section{Characterization of spectral convergence of Neumann LAPLACIAN}

In this section we give a necessary and sufficient condition for the convergence of the eigenvalues and eigenfunctions of the Laplace operator with Neumann boundary conditions. We refer to [1] and [3] for a general result in this direction, even in a more general context than the one in this note. In our particular case, we will consider the following situation: let $\Omega_{0}$ be a fixed bounded smooth (Lipschitz is enough) open set in $\mathbb{R}^{N}$ with $N \geq 2$ and let $\Omega_{\epsilon}$ be a family of domains such that for each fixed $0<\epsilon \leq \epsilon_{0}, \Omega_{\epsilon}$ is bounded and smooth with $\Omega_{0} \subset \Omega_{\epsilon}$.

Let us define now what we mean by the spectral convergence. For $0 \leq$ $\epsilon \leq \epsilon_{0}$, we denote by $\left\{\lambda_{n}^{\epsilon}\right\}_{n=1}^{\infty}$ the sequence of eigenvalues of the Neumann Laplacian in $\Omega_{\epsilon}$, always ordered and counting its multiplicity, and we denote by $\left\{\phi_{n}^{\epsilon}\right\}_{n=1}^{\infty}$ a corresponding set of orthonormal eigenfunctions in $\Omega_{\epsilon}$. Also, 
since we are considering domains which vary with the parameter $\epsilon$ and we will need to compare functions defined in $\Omega_{0}$ and in $\Omega_{\epsilon}$, we introduce the following space $H_{\epsilon}^{1}=H^{1}\left(\Omega_{0}\right) \oplus H^{1}\left(\Omega_{\epsilon} \backslash \bar{\Omega}_{0}\right)$, that is $\chi \in H_{\epsilon}^{1}$ if $\chi_{\mid \Omega_{0}} \in H^{1}\left(\Omega_{0}\right)$ and $\chi_{\mid\left(\Omega_{\epsilon} \backslash \bar{\Omega}_{0}\right)} \in H^{1}\left(\Omega_{\epsilon} \backslash \bar{\Omega}_{0}\right)$, with the norm

$$
\|\chi\|_{H_{\epsilon}^{1}}^{2}=\|\chi\|_{H^{1}\left(\Omega_{0}\right)}^{2}+\|\chi\|_{H^{1}\left(\Omega_{\epsilon} \backslash \bar{\Omega}_{0}\right)}^{2} .
$$

We have that $H^{1}\left(\Omega_{\epsilon}\right) \hookrightarrow H_{\epsilon}^{1}$ and in a natural way we have that if $\chi \epsilon$ $H^{1}\left(\Omega_{0}\right)$ via the extension by zero outside $\Omega_{0}$ we have $\chi \in H_{\epsilon}^{1}$. Hence, with certain abuse of notation we may say that if $\chi_{\epsilon} \in H_{\epsilon}^{1}, 0 \leq \epsilon \leq \epsilon_{0}$, then $\chi_{\epsilon} \stackrel{\epsilon \rightarrow 0}{\longrightarrow} \chi_{0}$ in $H_{\epsilon}^{1}$ if $\left\|\chi_{\epsilon}-\chi_{0}\right\|_{H^{1}\left(\Omega_{0}\right)}+\left\|\chi_{\epsilon}\right\|_{H^{1}\left(\Omega_{\epsilon} \backslash \Omega_{0}\right)} \stackrel{\epsilon \rightarrow 0}{\longrightarrow} 0$.

Definition 2.1. We will say that the family of domains $\Omega_{\epsilon}$ converges spectrally to $\Omega_{0}$ as $\epsilon \rightarrow 0$ if the eigenvalues and eigenprojectors of the Neumann Laplacian behave continuously at $\epsilon=0$. That is, for any fixed $n \in \mathbb{N}$ we have that $\lambda_{n}^{\epsilon} \rightarrow \lambda_{n}^{0}$ as $\epsilon \rightarrow 0$, and for each $n \in \mathbb{N}$ such that $\lambda_{n}^{0}<\lambda_{n+1}^{0}$ the spectral projections $P_{n}^{\epsilon}: L^{2}\left(\mathbb{R}^{N}\right) \rightarrow H^{1}\left(\Omega_{\epsilon}\right), P_{n}^{\epsilon}(\psi)=\sum_{i=1}^{n}\left(\phi_{i}^{\epsilon}, \psi\right)_{L^{2}\left(\Omega_{\epsilon}\right)} \phi_{i}^{\epsilon}$, satisfy

$$
\sup \left\{\left\|P_{n}^{\epsilon}(\psi)-P_{n}^{0}(\psi)\right\|_{H_{\epsilon}^{1}}, \psi \in L^{2}\left(\mathbb{R}^{N}\right),\|\psi\|_{L^{2}\left(\mathbb{R}^{N}\right)}=1\right\} \stackrel{\epsilon \rightarrow 0}{\longrightarrow} 0 .
$$

The convergence of the spectral projections is equivalent to the following: for each sequence $\epsilon_{k} \rightarrow 0$ there exists a subsequence, that we denote again by $\epsilon_{k}$ and a complete system of orthonormal eigenfunctions of the limiting problem $\left\{\phi_{n}^{0}\right\}_{n=1}^{\infty}$ such that $\left\|\phi_{n}^{\epsilon_{k}}-\phi_{n}^{0}\right\|_{H_{\epsilon_{k}}^{1}} \rightarrow 0$ as $k \rightarrow \infty$.

In order to write down the characterization, we need to consider the following quantity

$$
\tau_{\epsilon}=\min _{\substack{\phi \in H^{1}\left(\Omega_{\epsilon}\right) \\ \phi=0 \text { in } \Omega_{0}}} \frac{\int_{\Omega_{\epsilon}}|\nabla \phi|^{2}}{\int_{\Omega_{\epsilon}}|\phi|^{2}} .
$$

Observe that $\tau_{\epsilon}$ is the first eigenvalue of the following problem with a combination of Dirichlet and Neumann boundary conditions:

$$
\left\{\begin{aligned}
-\Delta u & =\tau u, & & \Omega_{\epsilon} \backslash \bar{\Omega}_{0}, \\
u & =0, & & \partial \Omega_{0}, \\
\frac{\partial u}{\partial n} & =0, & & \partial \Omega_{\epsilon} \backslash \partial \Omega_{0} .
\end{aligned}\right.
$$

We can prove the following,

Proposition 2.2. A necessary and sufficient condition for the spectral convergence of $\Omega_{\epsilon}$ to $\Omega_{0}$ is

$$
\tau_{\epsilon} \stackrel{\epsilon \rightarrow 0}{\longrightarrow}+\infty \text {. }
$$

We refer to [1] and [3] for a proof of this result.

Remark 2.3. The fact that $\Omega_{0} \subset \Omega_{\epsilon}$ can be relaxed. It is enough asking that for each compact set $K \subset \Omega_{0}$ there exists $\epsilon(K)$ such that $K \subset \Omega_{\epsilon}$ for $0<\epsilon \leq \epsilon(K)$, see [3]. 


\section{Measure COnVERGence OF the Domains}

In this section we provide an answer to the first question. Observe that in Proposition 2.2 we do not require that $\left|\Omega_{\epsilon} \backslash \Omega_{0}\right| \stackrel{\epsilon \rightarrow 0}{\longrightarrow} 0$. However, we have the following

Corollary 3.1. In the situation above if $\Omega_{\epsilon}$ converges spectrally to $\Omega_{0}$, then necessarily $\left|\Omega_{\epsilon} \backslash \Omega_{0}\right| \stackrel{\epsilon \rightarrow 0}{\longrightarrow} 0$.

Proof. This result is proved in [3] but for the sake of completeness and since it is a simple proof, we include it in here.

If this were not true then we will have a positive $\eta>0$ and a sequence $\epsilon_{k} \rightarrow 0$ such that $\left|\Omega_{\epsilon_{k}} \backslash \Omega_{0}\right| \geq \eta$. Let $\rho=\rho(\eta)$ be a small number such that $\mid\{x \in$ $\left.\mathbb{R}^{N} \backslash \Omega_{0}, \operatorname{dist}\left(x, \Omega_{0}\right) \leq \rho\right\} \mid \leq \eta / 2$. This implies that $\mid\left\{x \in \Omega_{\epsilon_{k}}, \operatorname{dist}\left(x, \Omega_{0}\right) \geq\right.$ $\rho\} \mid \geq \eta / 2$. Let us construct a smooth function $\gamma$ with $\gamma=0$ in $\Omega_{0}$, and $\gamma(x)=1$ for $x \in \mathbb{R}^{N} \backslash \Omega_{0}$ with $\operatorname{dist}\left(x, \Omega_{0}\right) \geq \rho$. Then obviously $\gamma \in H^{1}\left(\Omega_{\epsilon_{k}}\right)$ with $\|\nabla \gamma\|_{L^{2}\left(\Omega_{\epsilon_{k}}\right)} \leq C$ and $\|\gamma\|_{L^{2}\left(\Omega_{\epsilon_{k}}\right)} \geq(\eta / 2)^{\frac{1}{2}}$. This implies that $\tau_{\epsilon_{k}}$ is bounded. Hence it is not true that $\tau_{\epsilon} \stackrel{\epsilon \rightarrow 0}{\longrightarrow}+\infty$ and therefore, from Proposition 2.2 , we do not obtain the spectral convergence.

In particular, this result implies that the answer to question (Q1) is affirmative. That is, if we have the convergence of Neumann eigenvalues and eigenfunctions, necessarily we have that $\left|\Omega_{\epsilon} \backslash \Omega_{0}\right| \stackrel{\epsilon \rightarrow 0}{\longrightarrow} 0$.

\section{Distance CONVERGEnCE OF The DOMAins}

In this section we will provide an answer to question (Q2) and, as a matter of fact, we will see that the answer is No. We will prove this by constructing an example of a fixed domain $\Omega_{0}$ and a sequence of domains $\Omega_{\epsilon}$ with $\Omega_{0} \subset \Omega_{\epsilon}$ with the property that $\operatorname{dist}\left(\Omega_{\epsilon}, \Omega_{0}\right)$ does not converges to 0 , but the eigenvalues and eigenfunctions of the Laplace operator with Neumann boundary conditions in $\Omega_{\epsilon}$ converge to the ones in $\Omega_{0}$, see Definition 2.1.

As a matter of fact in [3, Section 5.2] a very particular example of a dumbbell domain (two disconnected domains joined by a thin channel) is provided so that the eigenvalues from the dumbbell converge to the eigenvalues of the two disconnected domains and no spectral contribution from the channel is observed. In this note we will obtain a family of channels for which the same phenomena occurs, see Corollary 4.4, and will provide a proof, different from the one given in 3 .

Let us consider a fixed domain $\Omega_{0} \subset \mathbb{R}^{N}$ which satisfies that $\Omega_{0} \subset\{x \in$ $\left.\mathbb{R}^{N}, x_{1}<0\right\}$ and such that

$$
\begin{aligned}
\Omega_{0} \cap\left\{x=\left(x_{1}, x^{\prime}\right) \in \mathbb{R} \times \mathbb{R}^{N-1},-1<x_{1}<1,\left|x^{\prime}\right| \leq \rho\right\} \\
\quad=\left\{x=\left(x_{1}, x^{\prime}\right) \in \mathbb{R} \times \mathbb{R}^{N-1},-1<x_{1}<0,\left|x^{\prime}\right| \leq \rho\right\}
\end{aligned}
$$

for some fixed $\rho>0$.

We will construct $\Omega_{\epsilon}$ as $\Omega_{\epsilon}=\operatorname{int}\left(\bar{\Omega}_{0} \cup \bar{R}_{\epsilon}\right)$, where $R_{\epsilon}$ is given as follows

$$
R_{\epsilon}=\left\{\left(x_{1}, x^{\prime}\right) \in \mathbb{R} \times \mathbb{R}^{N-1}: 0<x_{1}<L,\left|x^{\prime}\right|<g_{\epsilon}\left(x_{1}\right)\right\}
$$


where the function $g_{\epsilon}$ will be chosen so that $g_{\epsilon}>0, g_{\epsilon} \in C^{1}([0, L])$ and $g_{\epsilon} \rightarrow 0$ uniformly on $[0, L]$, see Figure 1, For the sake of notation we denote by $\Gamma_{0}^{\epsilon}=\partial R_{\epsilon} \cap\left\{x_{1}=0\right\}$ and $\Gamma_{L}^{\epsilon}=\partial R_{\epsilon} \cap\left\{x_{1}=L\right\}$.

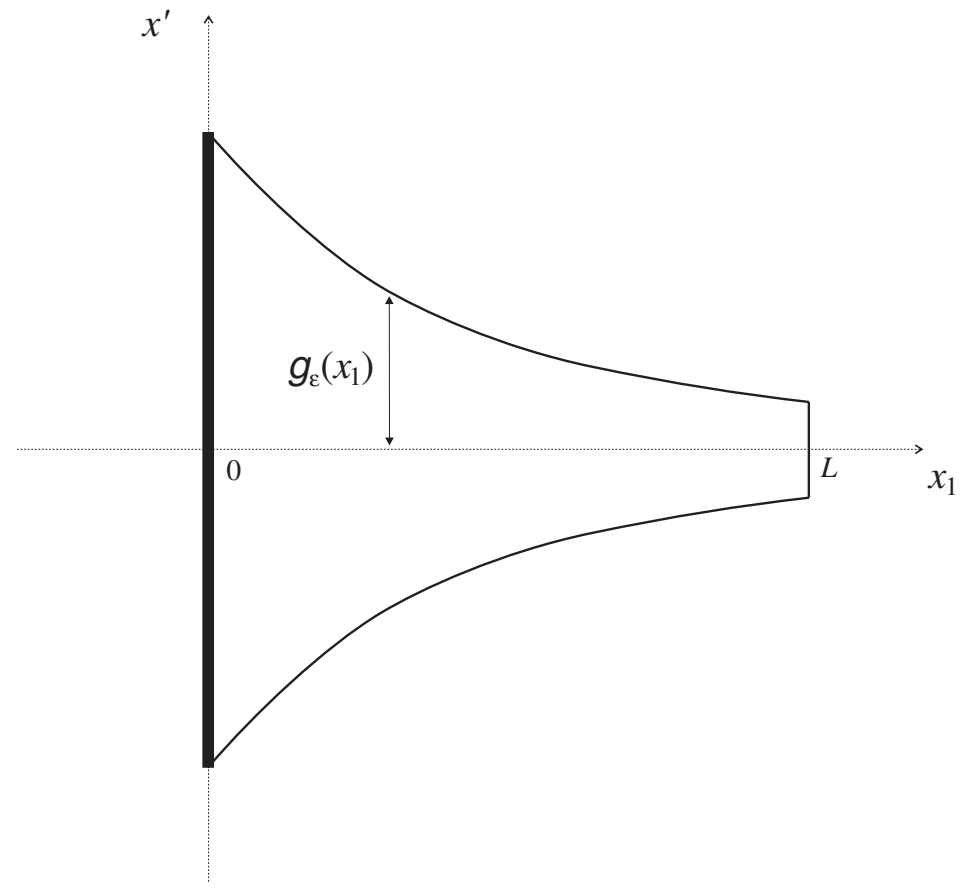

FiguRe 1. The exterior perturbation $R_{\epsilon}$. The thick line refers to the supplementary Dirichlet condition in the problem (4.2), while Neumann boundary conditions are imposed elsewhere.

We refer to 12 for a general reference on the behavior of solutions of partial differential equations on thin domains. See also the recent survey [7] for a study on the spectrum of the Laplacian on thin tubes in various settings, and for many related references.

Observe that if $L$ is fixed then $\operatorname{dist}\left(\Omega_{\epsilon}, \Omega_{0}\right)=L$ for each $0<\epsilon \leq \epsilon_{0}$. Moreover we will show that for certain choices of $g_{\epsilon}$ we obtain the spectral convergence of the Laplace operator. To prove this results, we use Proposition 2.2 and show that $\tau_{\epsilon} \rightarrow+\infty$. Notice that $\tau_{\epsilon}$, defined in (2.1) is the first eigenvalue of

$$
\left\{\begin{aligned}
-\Delta u & =\tau u, & & R_{\epsilon}, \\
u & =0, & & \Gamma_{0}^{\epsilon}, \\
\frac{\partial u}{\partial n} & =0, & & \partial R_{\epsilon} \backslash \Gamma_{0}^{\epsilon} .
\end{aligned}\right.
$$

Since we have Neumann boundary conditions on the lateral boundary of $R_{\epsilon}$, there clearly exist profiles of $g_{\epsilon}$ for which $\tau_{\epsilon}$ remains uniformly bounded as $\epsilon \rightarrow 0$. In fact, a simple trial-function argument shows that $\tau_{\epsilon} \leq \pi^{2} /(2 L)^{2}$ 
whenever $g_{\epsilon}(s) \geq g_{\epsilon}(0)$ for every $s \in[0, L]$. The idea to get $\tau_{\epsilon} \rightarrow+\infty$ consists in choosing a rapidly decreasing function $s \mapsto g_{\epsilon}(s)$, which enables one to get a large contribution to $\tau_{\epsilon}$ coming from the longitudinal energy due to the approaching Dirichlet and Neumann boundary conditions in the limit $\epsilon \rightarrow 0$. Let us notice that a similar trick to employ the repulsive contribution of such a combination of the boundary conditions have been used recently in 10 to establish a Hardy-type inequality in a waveguide; see also 11 for eigenvalue asymptotics in narrow curved strips with combined Dirichlet and Neumann boundary conditions. In our case, we are able to show

Proposition 4.1. With the notations above, for any function $\gamma \in C^{2}([0, L])$ satisfying

$$
0<\alpha_{0} \leq \gamma \leq \alpha_{1}<1, \quad \dot{\gamma}(L) \leq 0, \quad \text { and } \quad \ddot{\gamma} \geq \alpha_{2}>0
$$

for some positive numbers $\alpha_{0}, \alpha_{1}$ and $\alpha_{2}$, if we define $g_{\epsilon}=\gamma^{1 / \epsilon}$ we have that $\tau_{\epsilon} \stackrel{\epsilon \rightarrow 0}{\longrightarrow} 0$.

In particular, applying Proposition 2.2 we obtain the convergence of the eigenvalues and eigenfunctions of the Neumann Laplacian in $\Omega_{\epsilon}$ to the ones in $\Omega_{0}$.

Remark 4.2. Observe that a function $\gamma$ satisfying (4.3) necessarily satisfies that $\dot{\gamma}(s)<0$ for $0 \leq s<L$. Hence, the function $\gamma$ is decreasing.

Proof: Since $\tau_{\epsilon}$ is given by minimization of the Rayleigh quotient,

$$
\tau_{\epsilon}=\inf _{\substack{\phi \in H^{1}\left(R_{\epsilon}\right) \\ \phi=0 \text { in } \Gamma_{0}^{\epsilon}}} \frac{\int_{R_{\epsilon}}|\nabla \phi|^{2}}{\int_{R_{\epsilon}}|\phi|^{2}}
$$

we analyze the integral $\int_{R_{\epsilon}}|\nabla \phi|^{2}$ for a smooth real-valued function $\phi$ with $\phi=0$ in a neighborhood of $\Gamma_{0}^{\epsilon}$. We have

$$
\int_{R_{\epsilon}}|\nabla \phi|^{2}=\int_{0}^{L} \int_{\left|x^{\prime}\right|<g_{\epsilon}\left(x_{1}\right)}\left(\left|\phi_{x_{1}}\right|^{2}+\left|\nabla_{x^{\prime}} \phi\right|^{2}\right) d x^{\prime} d x_{1}
$$

Considering the change of variables $x_{1}=y_{1}, x^{\prime}=g_{\epsilon}\left(y_{1}\right) y^{\prime}$ which transforms $\left(x_{1}, x^{\prime}\right) \in R_{\epsilon}$ into $\left(y_{1}, y^{\prime}\right) \in Q$ where $Q$ is the cylinder $Q=\left\{\left(y_{1}, y^{\prime}\right): 0<\right.$ $\left.y_{1}<L,\left|y^{\prime}\right|<1\right\}$ and performing this change of variables in the integral above, elementary calculations show that

$$
\int_{R_{\epsilon}}|\nabla \phi|^{2}=\int_{Q}\left[\left(\varphi_{y_{1}}-\frac{\dot{g}_{\epsilon}}{g_{\epsilon}} \sum_{i=2}^{N} y_{i} \varphi_{y_{i}}\right)^{2}+\frac{1}{g_{\epsilon}^{2}} \sum_{i=2}^{N}\left|\varphi_{y_{i}}\right|^{2}\right] g_{\epsilon}^{N-1} d y
$$

where $\varphi(y)=\phi\left(y_{1}, g_{\epsilon}\left(y_{1}\right) y^{\prime}\right)$.

Writing the above expression in terms of the new function $\psi(y)=g_{\epsilon}\left(y_{1}\right)^{\frac{N-1}{2}} \varphi(y)$ so that

$$
\begin{aligned}
& g_{\epsilon}^{(N-1) / 2} \varphi_{y_{i}}=\psi_{y_{i}}, \quad i=2, \ldots, N, \\
& g_{\epsilon}^{(N-1) / 2} \varphi_{y_{1}}=-\frac{N-1}{2} \frac{\dot{g}_{\epsilon}}{g_{\epsilon}} \psi+\psi_{y_{1}},
\end{aligned}
$$


we get,

$$
\begin{aligned}
& \int_{R_{\epsilon}}|\nabla \phi|^{2} \\
&=\int_{Q}\left[\left(-\frac{N-1}{2} \frac{\dot{g}_{\epsilon}}{g_{\epsilon}} \psi+\psi_{y_{1}}-\frac{\dot{g}_{\epsilon}}{g_{\epsilon}} \sum_{i=2}^{N} y_{i} \psi_{y_{i}}\right)^{2}+\frac{1}{g_{\epsilon}^{2}} \sum_{i=2}^{N}\left|\psi_{y_{i}}\right|^{2}\right] d y \\
&=\int_{Q}\left[\left(-\frac{N-1}{2} \frac{\dot{g}_{\epsilon}}{g_{\epsilon}} \psi\right)^{2}+\left(\psi_{y_{1}}-\frac{\dot{g}_{\epsilon}}{g_{\epsilon}} \sum_{i=2}^{N} y_{i} \psi_{y_{i}}\right)^{2}-(N-1) \frac{\dot{g}_{\epsilon}}{g_{\epsilon}} \psi \psi_{y_{1}}\right. \\
&\left.\quad+(N-1) \frac{\dot{g}_{\epsilon}^{2}}{g_{\epsilon}^{2}} \sum_{i=2}^{N} y_{i} \psi_{y_{i}} \psi+\frac{1}{g_{\epsilon}^{2}} \sum_{i=2}^{N}\left|\psi_{y_{i}}\right|^{2}\right] d y \\
& \geq \int_{Q}\left[\left(\frac{N-1}{2}\right)^{2} \frac{\dot{g}_{\epsilon}^{2}}{g_{\epsilon}^{2}} \psi^{2}-(N-1) \frac{\dot{g}_{\epsilon}}{g_{\epsilon}} \psi \psi_{y_{1}}\right. \\
&\left.\quad+(N-1) \frac{\dot{g}^{2}}{g_{\epsilon}^{2}} \sum_{i=2}^{N} y_{i} \psi_{y_{i}} \psi+\frac{1}{g_{\epsilon}^{2}} \sum_{i=2}^{N} \psi_{y_{i}}^{2}\right] d y
\end{aligned}
$$

where we have used that $\left(\psi_{y_{1}}-\sum_{i=2}^{N} y_{i} \psi_{y_{i}} \frac{\dot{g}_{\epsilon}}{g_{\epsilon}}\right)^{2} \geq 0$. Via integration by parts in the second and third term above, we get,

$$
\begin{aligned}
\int_{Q}- & (N-1) \frac{\dot{g}_{\epsilon}}{g_{\epsilon}} \psi \psi_{y_{1}} d y=\int_{\left|y^{\prime}\right|<1} \int_{0}^{L}-(N-1) \frac{\dot{g}_{\epsilon}}{2 g_{\epsilon}}\left(\psi^{2}\right)_{y_{1}} d y_{1} d y^{\prime} \\
& =\int_{\left|y^{\prime}\right|<1}\left(-\left[(N-1) \frac{\dot{g}_{\epsilon}}{2 g_{\epsilon}} \psi^{2}\right]_{y_{1}=0}^{y_{1}=L}+\int_{0}^{L}(N-1)\left(\frac{\dot{g}_{\epsilon}}{2 g_{\epsilon}}\right) \psi^{2} d y_{1}\right) d y^{\prime} \\
& =-\int_{\left|y^{\prime}\right|<1}(N-1) \frac{\dot{g}_{\epsilon}(L)}{2 g_{\epsilon}(L)} \psi^{2}\left(L, y^{\prime}\right) d y^{\prime}+\int_{Q} \frac{N-1}{2}\left(\frac{\ddot{g}_{\epsilon}}{g_{\epsilon}}-\frac{\dot{g}_{\epsilon}^{2}}{g_{\epsilon}^{2}}\right) \psi^{2} d y
\end{aligned}
$$

and

$$
\begin{array}{r}
\int_{Q}(N-1) \frac{\dot{g}_{\epsilon}^{2}}{g_{\epsilon}^{2}} \sum_{i=2}^{N} y_{i} \psi_{y_{i}} \psi d y=\int_{0}^{L}(N-1) \frac{\dot{g}_{\epsilon}^{2}}{g_{\epsilon}^{2}} \sum_{i=2}^{N} \int_{\left|y^{\prime}\right|<1} y_{i} \frac{1}{2}\left(\psi^{2}\right)_{y_{i}} d y^{\prime} d y_{1} \\
=\int_{0}^{L} \frac{N-1}{2} \frac{\dot{g}_{\epsilon}^{2}}{g_{\epsilon}^{2}}\left(\int_{\left|y^{\prime}\right|=1} \psi^{2}-(N-1) \int_{\left|y^{\prime}\right|<1} \psi^{2} d y^{\prime}\right) d y_{1} .
\end{array}
$$

Hence if we require that $\dot{g}_{\epsilon}(L) \leq 0$, we have,

$$
\begin{gathered}
\int_{R_{\epsilon}}|\nabla \phi|^{2} \geq \int_{Q}\left[\frac{N-1}{2} \frac{\ddot{g}_{\epsilon}}{g_{\epsilon}}-\left(\left(\frac{N-1}{2}\right)^{2}+\frac{N-1}{2}\right) \frac{\dot{g}_{\epsilon}^{2}}{g_{\epsilon}^{2}}\right] \psi^{2} d y \\
+\int_{0}^{L} \frac{N-1}{2} \frac{\dot{g}_{\epsilon}^{2}}{g_{\epsilon}^{2}}\left(\int_{\left|y^{\prime}\right|=1} \psi^{2} d y^{\prime}\right) d y_{1}+\int_{Q} \frac{1}{g_{\epsilon}^{2}} \sum_{i=2}^{N} \psi_{y_{i}}^{2} d y
\end{gathered}
$$


The last two terms in this expression can be written as

$$
\int_{0}^{L} \frac{1}{g_{\epsilon}^{2}\left(y_{1}\right)}\left(\int_{\left|y^{\prime}\right| \leq 1}\left|\nabla_{y^{\prime}} \psi\right|^{2}+\frac{N-1}{2} \dot{g}_{\epsilon}^{2}\left(y_{1}\right) \int_{\left|y^{\prime}\right|=1} \psi^{2}\right) d y_{1}
$$

and we have that

$$
\int_{\left|y^{\prime}\right| \leq 1}\left|\nabla_{y^{\prime}} \psi\right|^{2}+\frac{N-1}{2} \dot{g}_{\epsilon}^{2} \int_{\left|y^{\prime}\right|=1} \psi^{2} \geq \rho \int_{\left|y^{\prime}\right| \leq 1} \psi^{2}
$$

with $\rho=\rho\left(y_{1}\right)$ being the first eigenvalue of the problem

$$
\left\{\begin{aligned}
-\Delta_{y^{\prime}} \psi & =\rho \psi, & & \left|y^{\prime}\right|<1, \\
\frac{\partial \psi}{\partial n}+\frac{N-1}{2} \dot{g}_{\epsilon}^{2}\left(y_{1}\right) \psi & =0, & & \left|y^{\prime}\right|=1
\end{aligned}\right.
$$

where $n$ denotes the outward unit normal vector field to the $(N-2)$ dimensional unit sphere $S_{1}=\left\{y^{\prime} \in \mathbb{R}^{N-1}:\left|y^{\prime}\right|=1\right\}$.

We claim that if we denote by $\lambda(\eta)$ the first eigenvalue of

$$
\left\{\begin{aligned}
-\Delta_{y^{\prime}} \psi & =\lambda \psi, & & \left|y^{\prime}\right|<1 \\
\frac{\partial \psi}{\partial n}+\eta \psi & =0, & & \left|y^{\prime}\right|=1
\end{aligned}\right.
$$

we have that $\frac{\lambda(\eta)}{\eta} \rightarrow \frac{\left|S_{1}\right|}{\left|B_{1}\right|}$ as $\eta \rightarrow 0$, where $B_{1}$ is the $(N-1)$ dimensional unit ball and $S_{1}$ its surface, which satisfy $\left|S_{1}\right|=(N-1)\left|B_{1}\right|$. As a matter of fact by standard continuity result we know that $\lambda(\eta) \rightarrow 0$ and its eigenfunction $\psi_{\eta}$, which is radially symmetric, converges to the constant function $1 / \sqrt{\left|B_{1}\right|}$, which is the first eigenfunction of the Neumann eigenvalue problem. But

$$
\lambda(\eta)=\int_{B_{1}}\left|\nabla_{y^{\prime}} \psi_{\eta}\right|^{2}+\eta \int_{S_{1}}\left|\psi_{\eta}\right|^{2} \geq \eta \int_{S_{1}}\left|\psi_{\eta}\right|^{2}
$$

which implies that

$$
\frac{\lambda(\eta)}{\eta} \geq \int_{S_{1}}\left|\psi_{\eta}\right|^{2} \rightarrow \frac{\left|S_{1}\right|}{\left|B_{1}\right|}
$$

Moreover, using $\psi=1 / \sqrt{\left|B_{1}\right|}$ as a test function in the Rayleigh quotient for $\lambda(\eta)$, we immediately obtain $\lambda(\eta) \leq \eta \frac{\left|S_{1}\right|}{\left|B_{1}\right|}$. This proves our claim. In particular, given $\delta>0$ small, we can choose $\eta_{0}=\eta_{0}(\delta)$ such that $\lambda(\eta)>$ $(N-1-\delta) \eta$ for $0<\eta \leq \eta_{0}$.

Therefore, if we choose the function $g_{\epsilon}$ such that $\dot{g}_{\epsilon}\left(y_{1}\right) \rightarrow 0$ uniformly in $y_{1} \in[0, L]$, we have that $\rho\left(y_{1}\right) \geq \frac{(N-1)(N-1-\delta)}{2} \dot{g}_{\epsilon}^{2}\left(y_{1}\right)$ for $\epsilon$ small enough.

Hence,

$$
\begin{aligned}
\int_{R_{\epsilon}}|\nabla \phi|^{2} \geq & \int_{Q}\left\{\frac{N-1}{2} \frac{\ddot{g}_{\epsilon}}{g_{\epsilon}}-\left[\left(\frac{N-1}{2}\right)^{2}\right.\right. \\
& \left.\left.-\frac{(N-1)(N-1-\delta)}{2}+\frac{N-1}{2}\right] \frac{\dot{g}_{\epsilon}^{2}}{g_{\epsilon}^{2}}\right\} \psi^{2} d y \\
= & \frac{N-1}{2} \int_{Q}\left\{\frac{\ddot{g}_{\epsilon}}{g_{\epsilon}}-\left[\frac{N-1}{2}-(N-1-\delta)+1\right] \frac{\dot{g}_{\epsilon}^{2}}{g_{\epsilon}^{2}}\right\} \psi^{2} d y
\end{aligned}
$$


and observe that the number $\kappa=\frac{N-1}{2}-(N-1-\delta)+1$ is strictly less than one for all values of $N \geq 2$ choosing a fixed and small $\delta>0$. If we denote by

$$
m_{\epsilon}=\inf _{0 \leq y_{1} \leq L}\left(\frac{\ddot{g}_{\epsilon}}{g_{\epsilon}}-\kappa \frac{\dot{g}_{\epsilon}^{2}}{g_{\epsilon}^{2}}\right)
$$

then

$$
\int_{R_{\epsilon}}|\nabla \phi|^{2} \geq \frac{N-1}{2} m_{\epsilon} \int_{Q} \psi^{2}=\frac{N-1}{2} m_{\epsilon} \int_{R_{\epsilon}} \phi^{2} .
$$

Consequently, $\tau_{\epsilon} \geq \frac{N-1}{2} m_{\epsilon}$.

Let us see that we can make a choice of the family of functions $g_{\epsilon}$, satisfying the two previous conditions we have imposed, that is $\dot{g}_{\epsilon}(L) \leq 0$ and $\dot{g}_{\epsilon}\left(y_{1}\right) \rightarrow 0$ uniformly in $0 \leq y_{1} \leq L$ such that $m_{\epsilon} \rightarrow+\infty$ as $\epsilon \rightarrow 0$.

Let us choose a function $\gamma \in C^{2}([0, L])$ satisfying (4.3) and let $g_{\epsilon}=\gamma^{1 / \epsilon}$. Then, we have

$$
\dot{g}_{\epsilon}=\frac{1}{\epsilon} \gamma^{\frac{1}{\epsilon}-1} \dot{\gamma}, \quad \ddot{g}_{\epsilon}=\frac{1}{\epsilon}\left(\frac{1}{\epsilon}-1\right) \gamma^{\frac{1}{\epsilon}-2} \dot{\gamma}^{2}+\frac{1}{\epsilon} \gamma^{\frac{1}{\epsilon}-1} \ddot{\gamma},
$$

and simple calculations show that

$$
\frac{\ddot{g}_{\epsilon}}{g_{\epsilon}}-\kappa \frac{\dot{g}_{\epsilon}^{2}}{g_{\epsilon}^{2}}=\left[\frac{1}{\epsilon}\left(\frac{1}{\epsilon}-1\right)-\kappa\left(\frac{1}{\epsilon}\right)^{2}\right]\left(\frac{\dot{\gamma}}{\gamma}\right)^{2}+\frac{\ddot{\gamma}}{\epsilon \gamma} \geq \frac{\alpha_{2}}{\alpha_{0}} \frac{1}{\epsilon}
$$

for $\epsilon>0$ small enough so that $\frac{1}{\epsilon}\left(\frac{1}{\epsilon}-1\right)-\kappa\left(\frac{1}{\epsilon}\right)^{2} \geq 0$. This shows that $m_{\epsilon} \rightarrow+\infty$ and it proves the proposition.

Remark 4.3. Now that we have been able to construct a thin domain $R_{\epsilon}$ as in (4.1) such that $\tau_{\epsilon} \stackrel{\epsilon \rightarrow 0}{\longrightarrow}+\infty$, we can construct another thin domain $\tilde{R}_{\epsilon}$ such that its "length" goes to infinity, its width goes to zero and still $\tilde{\tau}_{\epsilon} \stackrel{\epsilon \rightarrow 0}{\longrightarrow}+\infty$, where $\tilde{\tau}_{\epsilon}$ is the first eigenvalue of (4.2) in $\tilde{R}_{\epsilon}$ instead of $R_{\epsilon}$.

For this, let $R_{\epsilon}$ be a thin domain constructed as in Proposition 4.1 and let $\rho_{\epsilon}$ be a sequence with $\rho_{\epsilon} \rightarrow+\infty$ such that $\frac{\tau_{\epsilon}}{\rho_{\epsilon}^{2}} \rightarrow+\infty$ and $\alpha_{1}^{1 / \epsilon} \rho_{\epsilon} \rightarrow 0$. Define $\tilde{R}_{\epsilon}=\rho_{\epsilon} R_{\epsilon}$, that is

$$
\tilde{R}_{\epsilon}=\left\{\left(x_{1}, x^{\prime}\right): 0<x_{1}<\rho_{\epsilon} L,\left|x^{\prime}\right|<\rho_{\epsilon} g_{\epsilon}\left(x_{1}\right)\right\},
$$

then $0<\rho_{\epsilon} g_{\epsilon}\left(x_{1}\right) \leq \alpha_{1}^{1 / \epsilon} \rho_{\epsilon} \stackrel{\epsilon \rightarrow 0}{\longrightarrow} 0$ and $\tilde{\tau}_{\epsilon}=\frac{\tau_{\epsilon}}{\rho_{\epsilon}^{2}} \stackrel{\epsilon \rightarrow 0}{\longrightarrow}+\infty$.

Observe that if we require also a Dirichlet boundary condition in $\Gamma_{L}^{\epsilon}$, we can relax the conditions on $\gamma$ in Proposition 4.1 and in particular the condition $\dot{\gamma}(L) \leq 0$ can be dropped. Hence, we can show,

Corollary 4.4. With the notations above, for any function $\gamma \in C^{2}([0, L])$ satisfying

$$
0<\alpha_{0} \leq \gamma \leq \alpha_{1}<1, \quad \text { and } \quad \ddot{\gamma} \geq \alpha_{2}>0
$$


for some positive numbers $\alpha_{0}, \alpha_{1}$ and $\alpha_{2}$, if we define $g_{\epsilon}=\gamma^{1 / \epsilon}$ we have that $\tilde{\tau}_{\epsilon} \stackrel{\epsilon \rightarrow 0}{\longrightarrow} 0$, where $\tilde{\tau} \epsilon$ is the first eigenvalue of

$$
\left\{\begin{aligned}
-\Delta u & =\tau u, & & R_{\epsilon}, \\
u & =0, & & \Gamma_{0}^{\epsilon} \cup \Gamma_{L}^{\epsilon}, \\
\frac{\partial u}{\partial n} & =0, & & \partial R_{\epsilon} \backslash\left(\Gamma_{0}^{\epsilon} \cup \Gamma_{L}^{\epsilon}\right) .
\end{aligned}\right.
$$

Proof: This follows easily by a Neumann bracketing argument. More precisely, from the hypotheses, $\dot{\gamma}$ is a strictly increasing function. Hence, either $\gamma$ is strictly monotone in $(0, L)$, or there exists a unique $L^{*} \in(0, L)$ such that $\dot{\gamma}\left(L^{*}\right)=0$.

In the first case, if $\gamma$ is decreasing (respectively increasing) we substitute the Dirichlet boundary condition at $\Gamma_{L}^{\epsilon}$ (respectively at $\Gamma_{0}^{\epsilon}$ ) by a Neumann one. Then the new eigenvalue problem gives rise to $\tau_{\epsilon}$ defined exactly in the same way as (4.2) (modulo possibly a mirroring of $R_{\epsilon}$ ) and we have $\tilde{\tau}_{\epsilon} \geq \tau_{\epsilon} \rightarrow+\infty$ as $\epsilon \rightarrow 0$.

In the second case, we cut the domain $R_{\epsilon}$ in two domains $R_{\epsilon}^{0}=R_{\epsilon} \cap\{0<$ $\left.x_{1}<L^{*}\right\}, R_{\epsilon}^{1}=R_{\epsilon} \cap\left\{L^{*}<x_{1}<L\right\}$. We know that $\tilde{\tau}_{\epsilon} \geq \inf \left\{\tau_{\epsilon}^{0}, \tau_{\epsilon}^{1}\right\}$, where $\tau_{\epsilon}^{0}$ and $\tau_{\epsilon}^{1}$ are the corresponding eigenvalues in $R_{\epsilon}^{0}$ and $R_{\epsilon}^{1}$ with a Neumann boundary condition imposed at the newly created boundary $R_{\epsilon} \cap\left\{x_{1}=L^{*}\right\}$ on both domains. In both domains we can apply Proposition 4.1 as in the first case so that $\tau_{\epsilon}^{0}, \tau_{\epsilon}^{1} \stackrel{\epsilon \rightarrow 0}{\longrightarrow}+\infty$, which implies $\tilde{\tau}_{\epsilon} \rightarrow 0$.

Remark 4.5. This corollary recovers and generalizes the results from Section 5.2 in 3 .

\section{REFERENCES}

[1] J. M. Arrieta, Neumann eigenvalue problems on exterior perturbations of the domain, J. Differential Equations 118 (1995), 54-103.

[2] J. M. Arrieta, "Domain Dependence of Elliptic Operators in Divergence Form", Resenhas IME-USP, Vol. 3, No. 1, 107-123 (1997).

[3] J. M. Arrieta and A. N. Carvalho, Spectral convergence and nolinear dynamics of reaction diffusion equations under perturbations of the domain, J. Differential Equations 199 (1) (2004), 143-178.

[4] I. Babuška and R. Výborný, Continuous dependence of eigenvalues on the domains, Czech. Math. J. 15 (1965), 169-178.

[5] R. Courant and D. Hilbert, Methods of Mathematical Physics, Vol I, Wiley-Interscience, New York (1953). German Edition (1937).

[6] D. Daners, Dirichlet problems on varying domains, J. Differential Equations 188 (2003), 591-624.

[7] D. Grieser, Thin tubes in mathematical physics, global analysis and spectral geometry, Analysis on Graphs and its Applications (Cambridge, 2007), Proc. Sympos. Pure Math., vol. 77, pp. 565-594, Amer. Math. Soc., Providence, RI, 2008.

[8] A. Henrot, Extremum problems for eigenvalues of elliptic operators. Frontiers in Mathematics. Birkhäuser Verlag, Basel, 2006.

[9] D. Henry, Perturbation of the boundary in Partial Differential Equations, London Mathematical Society Lecture Note Series, 318, Cambridge University Press, (2005).

[10] H. Kovařík and D. Krejčiřík, A Hardy inequality in a twisted Dirichlet-Neumann waveguide, Math. Nachr. 281 (2008), no. 8, 1159-1168.

[11] D. Krejčiřík, Spectrum of the Laplacian in a narrow curved strip with combined Dirichlet and Neumann boundary conditions, ESAIM Control Optim. Calc. Var., to appear; Efirst on http://dx.doi.org/10.1051/cocv:2008035 
[12] G. Raugel, "Dynamics of Partial Differential Equations on Thin Domains", Lecture Notes in Mathematics 1609, Springer Verlag 1995, pp 208-315.

(J. M. Arrieta) Departamento de Matemática Aplicada, Universidad Complutense DE MADRID, 28040 MADRID SPAIN.

E-mail address: arrieta@mat.ucm.es

(D. Krejčiríík) Department of Theoretical Physics, Nuclear Physics Institute, Academy of Sciences, 25068 Řež, Czech Republic.

E-mail address: krejcirik@ujf.cas.cz 